\title{
Numerical Simulations on Mixing Dynamics of Spherical and Non-Spherical Tablets in a Pan Coater
}

\author{
Ram Chand \\ Physics Department, College of \\ Sciences, University of Hail \\ Hail, Saudi Arabia \\ ra.chand@uoh.edu.sa
}

Sujo Meghwar

Geography Department, Faculty of

Science, University of Sindh

Jamshoro, Pakistan

sujo@usindh.edu.pk

\author{
Ramzi Hadj Lajimi \\ Chemistry Department, College of \\ Sciences, University of Hail \\ Hail, Saudi Arabia \\ r.lajimi@uoh.edu.sa
}

Umer Farooq

Physics Department, College of

Sciences, University of Hail

Hail, Saudi Arabia

u.farooq@uoh.edu.sa

\author{
Saeed Uddin \\ National Center of Excellence in \\ Analytical Chemistry, Faculty of \\ Science, University of Sindh, Pakistan \\ chairman.bsek@hotmail.com \\ Najeh Rekik \\ Physics Department, College of \\ Sciences, University of Hail \\ Hail, Saudi Arabia \\ na.rekik@uoh.edu.sa
}

\begin{abstract}
Discrete element simulations provide valuable insight into the mixing dynamics of granular materials in industry. In this paper, numerical work is conducted in order to find the influence of pan rotational velocity and particle shape on mixing behavior. Four types of particles of different shapes were chosen: spherical, non-spherical type 1 (disk shape), non-spherical type 2 (capsule shape), and non-spherical type 3 (triangular shape). The pan mixer was filled with $\sim 30 \%$ volume of the same shape with the particles and was rotated at 15 RPM, 30 RPM, 45 RPM, and 60 RPM. The particles were colored as bottom-particles, middleparticles, and top-particles in order to visualize mixing efficiency. The homogeneity of the mixtures was determined by using contact dynamics of particles. The results show that fast-rotating pan (30-60 RPM) provides good mixing for all shapes of particles. However, non-spherical particles do not show as good mixing as spherical particles.
\end{abstract}

\section{Keywords- granular mixing; DEM; rotaing pan; tablet coating}

\section{INTRODUCTION}

Granular material is common in daily life [1-3], demonstrating a variety of complicated behaviors, some of which are not thoroughly perceived [3-7]. One of the issues in this material is mixing and de-mixing or segregation of granules [8-10]. Mixing and de-mixing of granular material with various properties is a strikingly testing procedure, pertinent to numerous mechanical applications [7-11]. In industry, particle mixing is an important process. Mixing and segregation phenomena describe an important issue into the process of mixing granular particles having various physical properties like size, density, shape, etc. Distinctly from liquids and gases, solid granules are not a thermal agitated system. In order to get a definite level of mixing they need to be mechanically activated. Traditionally, three mixing mechanisms are largely recognized: dispersion, convection, and shear mixing. Shear mixing is especially relevant to the granular material. It is a combination of both dispersion and convection mechanisms [12-13]. These mechanisms are performed in a solid system known as mixer. There are two types of mixers: tumbling mixers and agitated mixers [13]. In tumbling mixers, a completely confined container is rotated about an axis resulting in tumbling over of the granules in the mixture area inside the container. In contrast, convective mixers are characterized by the action of impellers in order to incorporate more shear into the mixture and provide a good mixing product in the end. In this study, pan coater mixer was used, which is a type of convective mixer, to study the mixing of tablet coating. In pharmaceutical industries, tablet coating is important for masking the unpleasant taste of a medicine, enhancing the appearance of the product, modifying its dissolution rate, etc. [14-20]. Many studies were conducted focusing on the mixing of grains with different sizes [12-13], but only a few studies considered shape and elongation, which are identified as important characteristics for inducing segregation [21-22]. The impact of different parameters on segregation is studied in [22]. The authors concluded that spherical grains have a higher flow-ability, leading to smaller repose angles and are more prone to segregation. Instead, irregular-shape particles are preventing them from free flowing and percolation $[13,17]$. The shape of granular particles has also a direct impact on the packing density of the granular media which is the main cause of segregation [12], but their work doesn't demonstrate the influence of the rotational speed of pan on non-spherical particles of complex shapes.

In this work, discrete element method (DEM) simulations are performed to study the mixing of binary mixtures of tablets having the same volume but different shapes. The mixing dynamics of a layered tablet was characterized in a rotating pan, highlighting the impact of the spherical and non-spherical shape, of the pan angular speed, and of contact dynamics between particles. Particle mixing is a significant but ineffectively comprehended part of coating of pharmaceutical tablets which is usually done in the rotating pan coaters [16]. 


\section{DEM SIMULATION AND RESULTS}

\section{A. Model Description}

DEM [23-25] has been increasingly applied for the investigation of tablet motion in coating mixers. In this method, Newtonian equations of motion are used to calculate particle motion and interactions. The force on each particle $i$ is calculated by the sum of gravitational and inter-particle components according to:

$$
\sum F_{i}=m_{i} g+F_{n}+F_{t}
$$

where $F_{i}$ and $F_{t}$ are normal and tangential components of force respectfully. The torque $\tau$ on each particle is the sum of the moment of the tangential forces $F_{t}$ arising from inter-particle contacts:

$$
\sum \tau_{i}=r_{i} \times F_{t}
$$

The normal forces $F_{n}$ and the tangential forces $F_{t}$ are calculated according to Hertzian contact model and MindlinDeresiewicz model respectively. Both normal and tangential force models are described by damping components where the damping coefficient is related to the coefficient of restitution as given in [26]. Accordingly, the normal force $F_{n}$ is given by:

$$
F_{n}=\frac{4}{3} E^{*} \sqrt{R^{*}} \delta_{n}^{\frac{3}{2}}
$$

where $E^{*}$ is the equivalent Young's Modulus, $R^{*}$ and the equivalent radius which are defined by:

$$
\begin{aligned}
& \frac{1}{E^{*}}=\frac{1-v_{i}^{2}}{E_{i}}+\frac{1-v_{j}^{2}}{E_{j}} \\
& \frac{1}{R^{*}}=\frac{1}{R_{i}}+\frac{1}{R_{j}}
\end{aligned}
$$

where $E_{i}, \vartheta_{i}, R_{i}$ and $E_{j}, \vartheta_{j}, R_{j}$ are the Young's Modulus, Poisson ratio and radius of particles $i$ and $j$ in contact.

These models are implemented in a commercially available DEM code called EDEM Academic 2.6.1 [27]. This code has already been validated for such types of particle flows and mixing in various conditions [28-29].

\section{B. Simulation Settings}

The DEM particles and the container are made of the materials defined in Table I. The container is modeled as a pan mixer by an open source mesh generator 3D application. The mesh cylinder has a total length of $16.6 \mathrm{~cm}$ along the axial direction with $20 \mathrm{~cm}$ diameter. In order to mix the particles, some baffles are created inside this pan. The schematic setup with all the measurements of the pan are given in Figure 1. After generating the geometry, this pan mesh is imported into EDEM code. We modeled 4 types of granular particles by EDEM code as shown in Figure 2.

First, we will initialize the spherical particles randomly by pouring from injector. The diameter of the particle is $3 \mathrm{~mm}$. We color them as red for bottom, green for middle and blue for top particles so that mixing can easily be deduced from their contact dynamics. In the same way, we will generate 3 other types of particles in the mixer. All other parameters of the particles are given in Table I. After pouring the particles of the same shape into the pan, the tablets in the $25 \%$-filled mixer were allowed to settle under the effect of gravity. After settling of all particles, the mixer pan is set to rotate with a rotational speed $\omega$ in the unit of revolution per minute (rpm or RMP). We set the rotational speed at $15 \mathrm{rpm}, 30 \mathrm{rpm}, 45 \mathrm{rpm}$, and $60 \mathrm{rpm}$ for 10000 s. Snapshots of the settled states and the mixed states are shown in Figure 3 for non-spherical type 2.

TABLE I. SIMULATION INPUT PARAMETERS

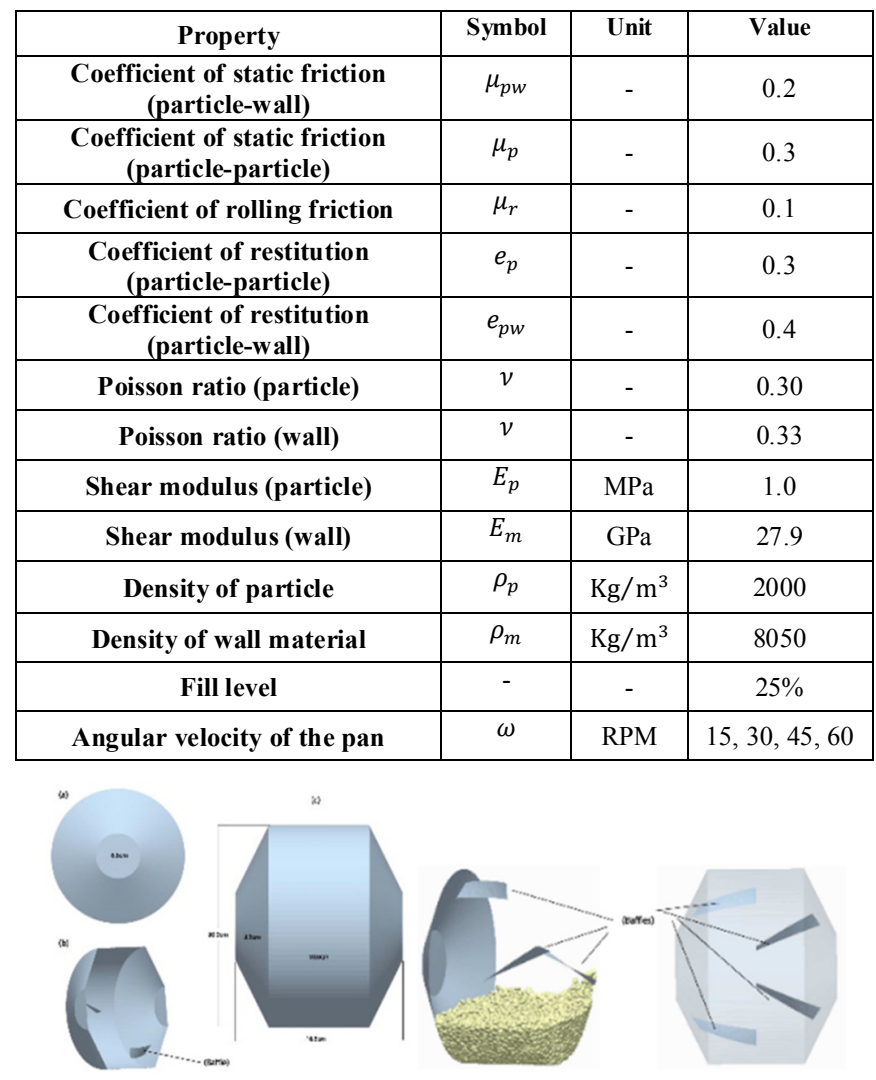

Fig. 1. Simulation setup: internal and external properties of mixing pan

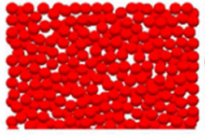

(a)

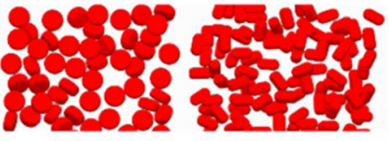

(c)

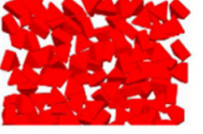

(d)
Fig. 2. Shapes of particles: (a) spherical, (b) disk-type (non-spherical type 1), (c) capsule (non-spherical type 2), (d) triagulate (non-spherical type 3 )
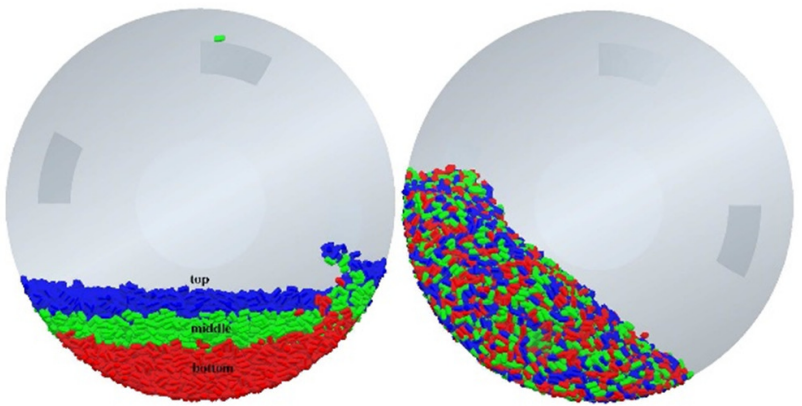

Fig. 3. Snapshot of mixing of non-spherical particles (before and after) 


\section{Mixing Behavior Deduced from Contact Number}

In order to measure the variance of the mixture, contact dynamics of particles was used. Note that initially the number of contacts among bottom, middle, and top was zero. After the mixer was rotated, the number of contacts increases and reaches a steady value, which indicates that the particles are fully mixed. After deducing the number of contacts, we can plot it for the various types of particles.

\section{Mixing Profile from the Rotating Pan}

Mixing profiles showing the contact dynamics of all types of particles are depicted in Figure 4, for all rotation velocities. In all mixing profiles, the study was focused to bottom-top layers of particles only. In the beginning, both layers were not in contact with each other, so the mixing state was zero. Once the mixing starts, the number of contact increases which can be clearly seen from the plots of Figure 4. All simulations were run for 1000 s duration, but the results show that steady state mixing was achieved after 30 s of rotation. Also, the results show that, slowly rotating pan takes a little bit longer time to achieve the same mixing state.
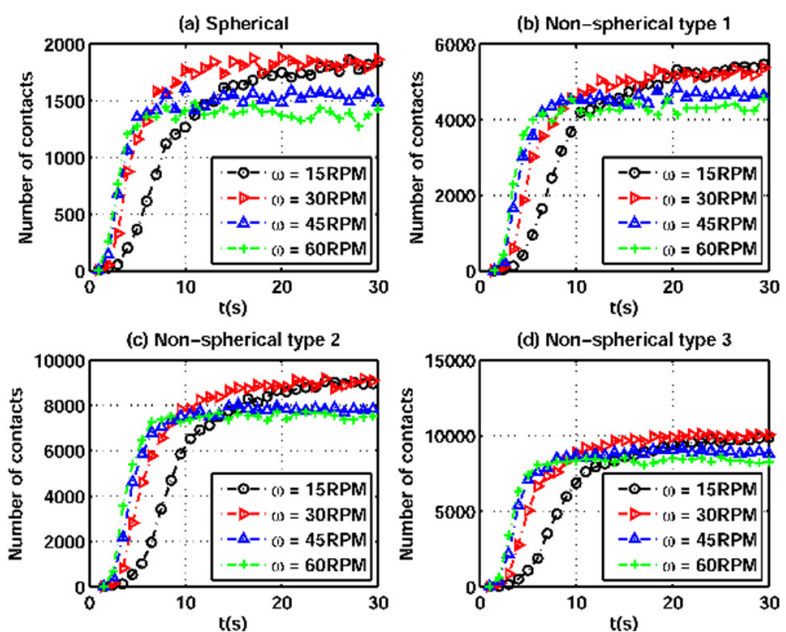

Fig. 4. Mixing of spherical and non-spherical particles (bottom-top layers)
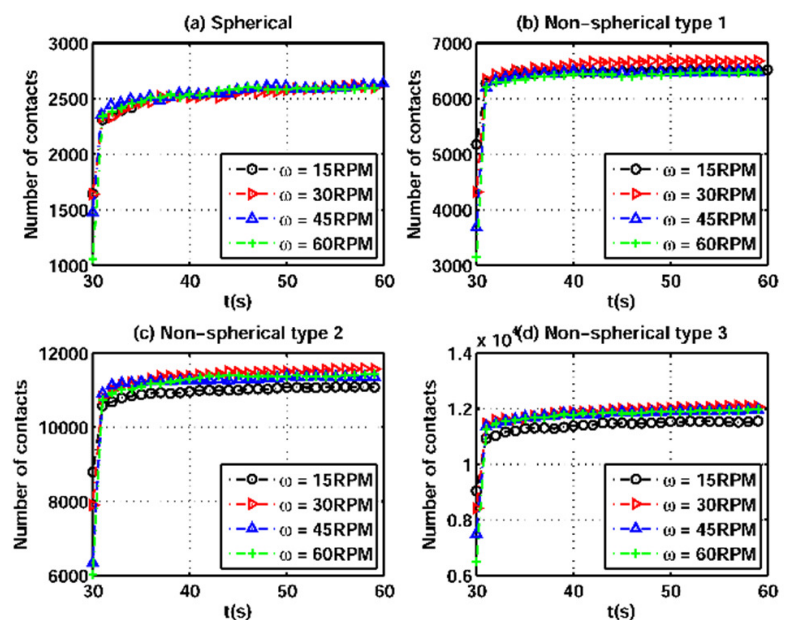

Fig. 5. Mixing profiles of spherical and non-spherical particles after rotations are completed (bottom-top layers)
The mixing profiles from 30 s to 60 s are shown in Figure 5. We see that steady states of mixing are achieved. For nonspherical particles, the slowly rotating pan doesn't exhibit good mixing state. The spherical particles mix repeatedly at all rotational speeds as plotted in Figure 6.

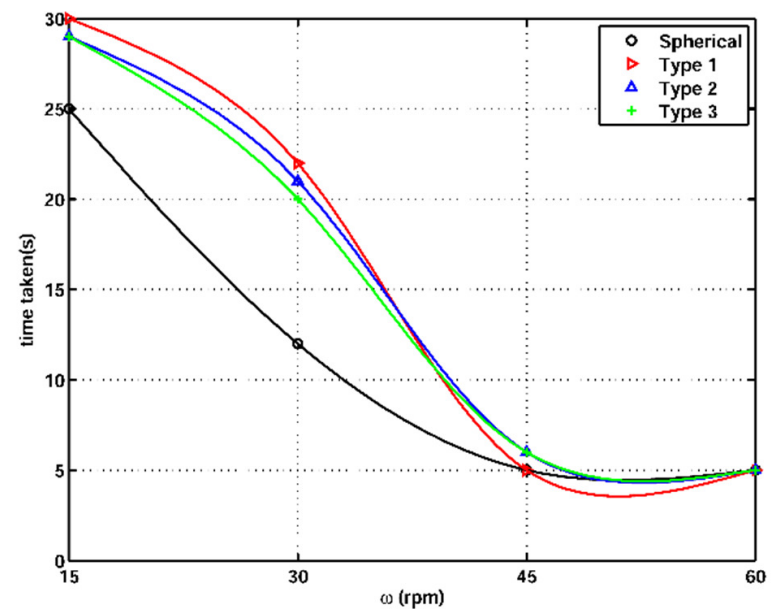

Fig. 6. Time taken for the final mixing for all rotational speeds

\section{E. Maximum Mixing}

In order to study the influence of angular velocity of the pan on the mixing of particles over time, we further define the parameter maximum mixing $\chi_{\max }$ as follows:

$$
\chi_{\max }=\frac{c}{c_{\max }}
$$

where $C$ is the contact number after the rotations are completed and $C_{\max }$ is the total number of contacts for a particular shape type particle. In Figure 7 the data taken from EDEM for maximum mixing $\chi_{\max }$ of all profiles are shown.

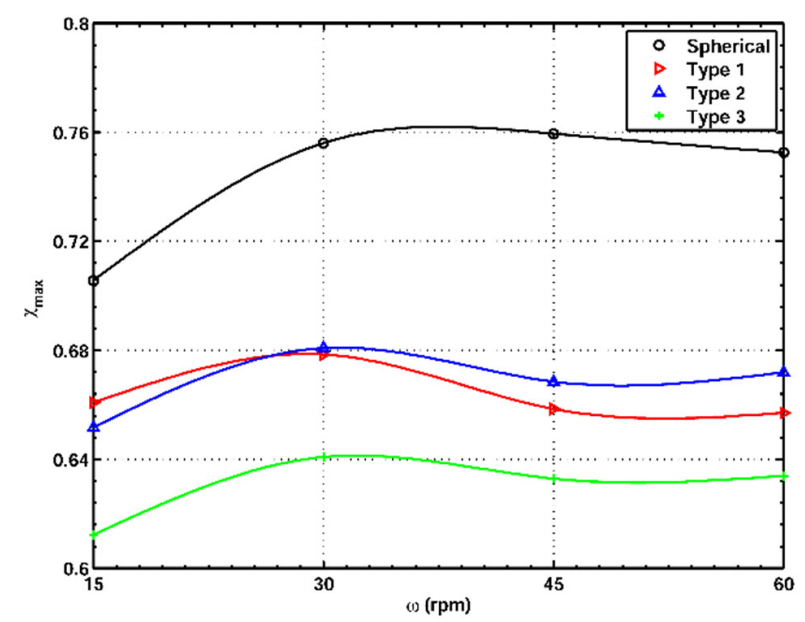

Fig. 7. Maximum mixing $\left(\chi_{\max }\right)$ achieved

The result shows that type 3 shape particles don't show good mixing as compared to spherical particles, because nonspherical particles reveal more opposition to motion and mix. The reason is that non-spherical shape creates irregular voids 
between the particles that prevent them from further mixing. Even though blades were used inside the pan to provide more shear to the particles, the voidage in non-spherical particles transfers a little shear to nearby particles [8, 11, 30-32]. Consequently, lower granular temperature is achieved which reduces the mixing $[8,11,31]$. Further work in this regard is needed to study the mixing mechanism of non-spherical granular particles mixed with fluids such as water.

\section{CONCLUSION}

In this work, DEM simulations were performed to study the influence of pan rotational velocity and the shape of granules on mixing behavior. Four types of particles having different shapes were poured. The pan mixer was filled with $\sim 30$ vol $\%$ of the same shape particles and was rotated at 15 RPM, 30 RPM, 45 RPM, and 60 RPM. The homogeneity of the mixtures was determined by using contact dynamics of particles. It has been found that fast rotating pan such as 30-60 RPM provides good mixing for all shapes of particles. The triangulate shape particles do not show good mixing while the spherical particle have the best mixing among all four types of particles studied in this work.

\section{ACKNOWLEDGMENT}

This research was funded by the Deanship of Scientific Research (DSR) of the University of Hail, Saudi Arabia (project number: 0150298).

\section{REFERENCES}

[1] R. D Mindlin, "Elastic spheres in contact under varying oblique forces", Journal of Applied Mechanics, Vol. 20, pp. 327-344, 1953

[2] R. Chand, Saeeduddin, M. A. Khaskheli, A. M. Soomro, H. Saleem, W. A. Bhutto, A. H. Nizamani, M. Y. Soomro, N. M. Shaikh, S. V. Muniandy, "Fractional analysis of light scattering data from gravitydriven granular flows", International Journal of Computer Science and Network Security, Vol. 19, No. 7, pp. 83-89, 2019

[3] R. Chand, A. Qadir, S. Qing-Fan, Z. Ning, S. Gang, "Anomalous increase of apparent mass in a silo due to percolation", Chinese Physics Letters, Vol. 28, No. 9, Article ID 098301, 2011

[4] A. Firoozfar, A. Rostami, H. Ghaderi, H. Zamani, A. Rostamkhani, "Assessing the effects of length, slope and distance between piles on the bearing capacity of a pile group under axial loading in granular soil", Engineering, Technology \& Applied Science Research, Vol. 7, No. 5, pp. 1894-1899, 2017

[5] R. Chand, Q. Shi, A. Qadir, S. Ma, G. Sun, "Fluctuations in apparent mass at the bottom of granular columns due to different arrangements", Physica A: Statistical Mechanics and its Applications, Vol. 391, No. 10, pp. 2936-2939, 2012

[6] R. Chand, M. A. Khaskheli, A. Qadir, Y. Sandali, Q. Shi, "Influence of spontaneous percolation on apparent mass at the bottom of a Janssen granular column", Physica A: Statistical Mechanics and its Applications, Vol. 393, pp. 96-100, 2014

[7] M. A. Soomro, A. S. Brohi, M. A. Soomro, D. K. Bangwar, S. A. Bhatti, "3D numerical modeling of pile group responses to excavation-induced stress release in silty clay", Engineering, Technology \& Applied Science Research, Vol. 8, No. 1, pp. 2577-2584, 2018

[8] R. Chand, S. V. Muniandy, C. S. Wong, J. Singh, "Discrete element method study of shear-driven granular segregation in a slowly rotating horizontal drum", Particuology, Vol. 32, pp. 89-94, 2017

[9] Y. Fan, K. M. Hill, "Phase transitions in shear-induced segregation of granular materials", Physical Review Letters, Vol. 106, No. 21, Article ID 218301, 2011

[10] M. K. Saeed, M. S. Siraj, "Mixing study of non-spherical particles using DEM", Powder Technology, Vol. 344, pp. 617-627, 2019
[11] R. Chand, M. A. Khaskheli, A Qadir, B. Ge, Q. Shi, "Discrete particle simulation of radial segregation in horizontally rotating drum: Effects of drum-length and non-rotating end-plates", Physica A: Statistical Mechanics and its Applications, Vol. 391, No. 20, pp. 4590-96, 2012

[12] A. Hassanpour, M. Pasha, M. Alizadeh, M. Ghadiri, A. Bayly, "The effect of particle shape on predicted segregation in binary powder mixtures", Powder Technology, Vol. 319, pp. 313-322, 2017

[13] C. Piacenza, M. Marconati, C. Hare, A. C. Santomaso, M. Ramaioli, "Mixing grains with different elongation in a rotating drums", available at: https://arxiv.org/abs/1809.00153, 2018

[14] J. Bridgwater, "Mixing of particles and powders: Where next?", Particuology, Vol. 8, No. 6, pp. 563-567, 2010

[15] J. Bridgwater, "Mixing of powders and granular materials by mechanical means-A perspective", Particuology, Vol. 10, No. 4, pp. 397-427, 2012

[16] E. Sahni, R. Yau, B. Chaudhuri, "Understanding granular mixing to enhance coating performance in a pan coater: Experiments and simulations", Powder Technology, Vol. 205, No. 1-3, pp. 231-241, 2011

[17] A. Dubey, R. Hsia, K. Saranteas, D. Brone, T, Misra, F. J. Muzzio, "Effect of speed, loading and spray pattern on coating variability in a pan coater", Chemical Engineering Science, Vol. 66, No. 21, pp. 51075115,2011

[18] G. R. Chandratilleke, A. B. Yu, J. Bridgwater, "A DEM study of the mixing of particles induced by a flat blade", Chemical Engineering Science, Vol. 79, pp. 54-74, 2012

[19] L. Obregon, A. Realpe, C. Rinaldi, C. Velazquez, "Mixing of granular materials, Part I: Effect of periodic shear", Powder Technology, Vol. 197, No. 1-2, pp. 9-16, 2010

[20] L. Obregon, A. Realpe, C. Velazquez, "Mixing of granular materials, Part II: Effect of particle size under periodic shear", Powder Technology, Vol. 201, No. 3, pp. 193-200, 2010

[21] C. Henrique, G. Batrouni, D. Bideau, "Diffusion as a mixing mechanism in granular materials", Physical Review E, Vol. 63, Article ID 011304, 2000

[22] Y. Fan, K. M. Hill, "Theory for shear-induced segregation of dense granular mixtures", New Journal of Physics, Vol. 13, Article ID 095009, 2011

[23] P. A. Cundall, O. D. L. Strack, "A discrete numerical model for granular assemblies", Geotechnique, Vol. 29, No. 1, pp. 47-65, 1979

[24] F. Bertrand, L. A. Leclaire, G. Levecque, "DEM-based models for the mixing of granular materials", Chemical Engineering Science, Vol. 60, No. 8-9, pp. 2517-2531, 2005

[25] M. Rajabi, H. S. Rad, M. S. Masoudian, "A numerical model for caprock analysis for subsurface gas storage applications", Engineering, Technology \& Applied Science Research, Vol. 8, No. 1, pp. 2438-2446, 2018

[26] Y. Tsuji, T. Tanaka, T. Ishida, "Lagrangian numerical simulation of plug flow of cohesionless particles in a horizontal pipe", Powder Technology, Vol. 71, No. 3, pp. 239-250, 1992

[27] www.edemsimulation.com

[28] Y. Wang, F. Tonon, "Dynamic validation of a discrete element code in modeling rock fragmentation", International Journal of Rock Mechanics and Mining Sciences, Vol. 48, No. 4, pp. 535-545, 2011

[29] C. P. Alonso, J. A. Delgadillo, "Experimental validation of 2D DEM code by digital image analysis in tumbling mills", Minerals Engineering, Vol. 25, No. 1, pp. 20-27, 2012

[30] M. Alian, F. E. Mozaffari, S. R. Upreti, J. Wu, "Using discrete element method to analyze the mixing of the solid particles in a slant cone mixer”, Chemical Engineering Reseach and Design, Vol. 93, pp. 318329,2015

[31] D. Nguyen, A. Rasmuson, I. N. Bjorn, K. Thalberg, "CFD simulation of transient particle mixing in a high shear mixer", Powder Technology, Vol. 258, pp. 324-330, 2014

[32] J. F. Camenen, Y. Descantes, "Geometrical properties of rigid frictionless granular packings as a function of particle size and shape", Physical Review E, Vol. 96, Article ID 012904, 2017 